\title{
Lipid Peroxidation End-Products as a Key of Oxidative Stress: Effect of Antioxidant on Their Production and Transfer of Free Radicals
}

\author{
Hanaa Ali Hassan Mostafa Abd El-Aal \\ Additional information is available at the end of the chapter
}

http://dx.doi.org/10.5772/45944

\section{Introduction}

\subsection{Oxidative stress}

The term oxidative stress; is a state of unbalanced tissue oxidation refers to a condition in which cells are subjected to excessive levels of molecular oxygen or its chemical derivatives called reactive oxygen species (ROS).Under physiological conditions, the molecular oxygen undergoes a series of reactions that ultimately lead to the generation of superoxide anion $\left(\mathrm{O}_{2}-\right)$, hydrogen peroxide $\left(\mathrm{H}_{2} \mathrm{O}_{2}\right)$ and $\mathrm{H}_{2} \mathrm{O}$. Peroxynitrite (OONO-), hypochlorus acid $(\mathrm{HOCl})$, the hydroxyl radical $(\mathrm{OH}$.$) , reactive aldehydes, lipid peroxides and nitrogen oxides$ are considered among the other oxidants that have relevance to vascular biology.

Oxygen is the primary oxidant in metabolic reactions designed to obtain energy from the oxidation of a variety of organic molecules. Oxidative stress results from the metabolic reactions that use oxygen, and it has been defined as a disturbance in the equilibrium status of pro-oxidant/anti-oxidant systems in intact cells. This definition of oxidative stress implies that cells have intact pro-oxidant/anti-oxidant systems that continuously generate and detoxify oxidants during normal aerobic metabolism. When additional oxidative events occur, the pro-oxidant systems outbalance the anti-oxidant, potentially producing oxidative damage to lipids, proteins, carbohydrates, and nucleic acids, ultimately leading to cell death in severe oxidative stress. Mild, chronic oxidative stress may alter the anti-oxidant systems by inducing or repressing proteins that participate in these systems, and by depleting cellular stores of anti-oxidant materials such as glutathione and vitamin E (Laval, 1996). Free radicals and other reactive species are thought to play an important role oxidative stress resulting in many human diseases. Establishing their precise role requires the ability to measure them and the oxidative damage that they cause (Halliwell and Whiteman, 2004). 
Oxidative stress is involved in the process of aging (Kregel and Zhang 2007) and various chronic diseases such as atherosclerosis (Fearon and Faux 2009), diabetes (Ceriello and Motz, 2004) and eye disease ( $\mathrm{Li}$ et al. 2009), whereas fruit and vegetable diets rich in antioxidants such as polyphenols, vitamin C, and carotenoids are correlated with a reduced risk of such chronic diseases (Dherani et al. 2008). An excessive amount of reactive oxygen/nitrogen species (ROS/RNS) leading to an imbalance between antioxidants and oxidants can cause oxidative damage in vulnerable targets such as unsaturated fatty acyl chains in membranes, thiol groups in proteins, and nucleic acid bases in DNA (Ceconi et al. 2003). Several assays to measure " total " antioxidant capacity of biological systems have been developed to investigate the involvement of oxidative stress in pathological conditions or to evaluate the functional bioavailability of dietary antioxidants. Conventional assays to determine antioxidant capacity primarily measure the antioxidant capacity in the aqueous compartment of plasma. Consequently, water soluble antioxidants such as ascorbic acid, uric acid, and protein thiols mainly influence these assays, whereas fat - soluble antioxidants such as tocopherols and carotenoids show little inf uence over the many results. However, there are new approaches to define the total antioxidant capacity of plasma, which reflect the antioxidant network between water - and fat - soluble antioxidants. Revelation of the mechanism of action of antioxidants and their true antioxidant potential can lead to identifying proper strategies to optimize the antioxidant defense systems in the body.

\subsection{Measurement of oxidative damage}

A basic approach to study oxidative stress would be to measure some products such as (i) free radicals; (ii) radical-mediated damages on lipids, proteins or DNA molecules; and iii) antioxidant enzymatic activity or concentration.

\subsubsection{Free radicals}

Free radicals are reactive compounds that are naturally produced in the human body. They can exert positive effects (e.g. on the immune system) or negative effects (e.g. lipids, proteins or DNA oxidation). Free radicals are normally present in the body in minute concentrations. Biochemical processes naturally lead to the formation of free radicals, and under normal circumstances the body can keep them in check. If there is excessive free radical formation, however, damage to cells and tissue can occur (Wilson, 1997). Free radicals are toxic molecules, may be derived from oxygen, which are persistently produced and incessantly attack and damage molecules within cells; most frequently, this damage is measured as peroxidized lipid products, protein carbonyl, and DNA breakage or fragmentation. Collectively, the process of free radical damage to molecules is referred to as oxidative stress (Reiter et al., 1997).To limit these harmful effects, an organism requires complex protection the antioxidant system. This system consists of antioxidant enzymes (catalase, glutathione peroxidase, superoxide dismutase) and non-enzymatic antioxidants (e.g. vitamin E [tocopherol], vitamin A [retinol], vitamin C [ascorbic acid], glutathione and uric acid). An 
imbalance between free radical production and antioxidant defence leads to an oxidative stress state, which may be involved in aging processes and even in some pathology (e.g. cancer and Parkinson's disease).

\subsubsection{Formation of free radicals}

Normally, bonds don't split in a way that leaves a molecule with an odd, unpaired electron. But when weak bonds split, free radicals are formed. Free radicals are very unstable and react quickly with other compounds, trying to capture the needed electron to gain stability. Generally, free radicals attack the nearest stable molecule, gaining its electron. When the "attacked" molecule loses its electron, it becomes a free radical itself, beginning a chain reaction. Once the process is started, it can cascade, finally resulting in the disruption of a living cell. Some free radicals arise normally during metabolism. Sometimes the body's immune system's cells purposefully create them to neutralize viruses and bacteria. However, environmental factors such as pollution, radiation, and toxins can also spawn free radicals. Normally, the body can handle free radicals, but if antioxidants are unavailable, or if the free-radical production becomes excessive, damage can occur. Of particular importance is that free radical damage accumulates with age (Packer, 1994).

\subsubsection{Sources of free radicals}

Free radicals have two principle sources: endogenous sources and exogenous sources. Endogenous sources of free radicals include those that are generated intracellularly, acting within the cell, and those that are formed within the cell, but are released into the surrounding area. These intracellular free radicals result from auto-oxidation and consequent inactivation of small molecules such as reduced thiols and flavins. They may also occur as a result of the activity of certain oxidases, lipoxygenases, cyclo-oxygenases, dehydrogenases and peroxidases. Electron transfer from metals such as iron to oxygencontaining molecules can also initiate free radical reactions paradoxically; antioxidants may also produce free radicals (Weir et al., 1996). A wide range of free radical molecular species are endogenous. The singlet oxygen is not a free radical but is nevertheless a reactive oxygen species and capable of causing tissue damage (Zebger et al., 2004). Exogenous sources of free radicals are environmental sources. Environmental sources of free radicals include exposure to ionizing radiation (from industry, sun exposure, cosmic rays, and medical X-rays), ozone and nitrous oxide (primarily from automobile exhaust), heavy metals (such as mercury, cadmium, and lead), cigarette smoke (both active and passive), alcohol, unsaturated fat, and other chemicals and compounds from food, water, and air. The exogenous sources of free radicals resulting from ionizing radiation play a major role in free radical production. The energy transferred into water from ionizing particles ionizes the water molecule. The water ions produced dissociate yielding free radicals (Valencia and Moran, 2004).

There are two enzymes including Aldehyd oxidase (AO) and xanthine oxidase $(\mathrm{XO})$, they have a very close evolutionary relationship, based on the recent cloning of the gens and they show a high degree of amino acid sequence homology (Terao et al., 2000). They have been 
suggested to be relevant to the pathophysiology of a number of clinical disorders (Wright et al., 1995). Aldehyd oxidase (AO) commonly exists in vertebrates. Although the liver is the main site for aldehyde oxidase this enzyme has also been reported in kidney, lung, muscle, spleen, stomach, heart and brain (Beedham, 2002). The enzyme in liver of various species catalyzes the oxidation of a number of aldehydes and nitrogenous and also catalyzes the metabolism of physiological compounds such as retinaldehyde and monoamine neurotransimeters (Huang and Ichikawa, 1994). Reduction of oxygen during substrate turnover, leads to the formation of superoxide anion and hydrogen peroxide as ROS. This capacity has attracted attention to the possible role of aldehyde oxidase as a source of ROS. In vivo, it seems that aldehyde oxidase together with cytochrome P450 are quantitative, the most important cellular sources for ROS (Al-Omar et al., 2004). Additionally, the most likely sources of free radicals are xanthine oxidase (XO) (McCord, 1985). This enzyme is high particularly in liver and intestine. Although XO generates ROS and evidence has been presented for its role in the development of ischaemic intestinal, hepatic and renal damage (Cohen, 1992). It may also contribute to the development of lung and myocardial reperfusion injury after ischaemic episodes.

\subsubsection{Production of free radicals}

Free radicals are produced in a number of ways in biological systems (Halliwell and Whiteman, 2004):

a. Exposure to ionizing radiation is a major cause of free radical production. When irradiated water is ionized, and electron is removed from the molecule, leaving behind an ionized water molecule. The damaging species resulting from the radiolysis of water are the free radicals $\bullet \mathrm{H}$ and $\bullet \mathrm{OH}$ and hydrated electrons. They are highly reactive and have a lifetime on the order of $10-9$ to $10-11$ seconds. The hydroxyl radical is extremely reactive and is carcinogenic. Since water presents the largest number of target molecules in a cell, most of the energy transfer goes on in water when a cell is irradiated, rather then the solute consisting of protein, carbohydrate, nucleic acid, and bioinorganic molecules. Oxygen is an excellent electron acceptor and can combine with the hydrogen radical to form a peroxyl radical. Hydrogen peroxide is toxic and when present in sufficient quantities can interfere with normal cellular metabolism.

b. Enzymes and transport molecules also generate free radicals as a normal consequence of their catalytic function.

c. Auto-oxidation reactions produce free radicals from the spontaneous oxidation of biological molecules involved in non-enzymatic electron transfers.

d. Physical exercise also increases oxidative stress and causes disruptions of the homeostasis. Training can have positive or negative effects on oxidative stress depending on training load, training specificity and the basal level of training. Moreover, oxidative stress seems to be involved in muscular fatigue and may lead to overtraining. 


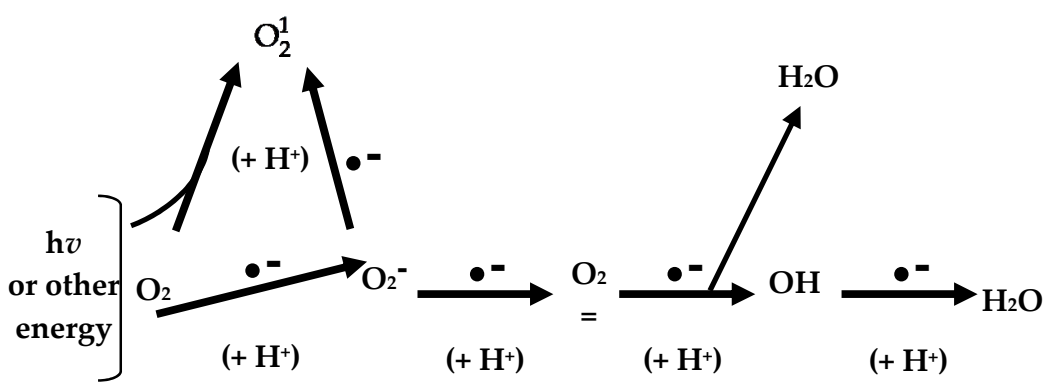

$\begin{array}{cccc}\begin{array}{c}\text { Molecular } \\ \text { oxygen }\end{array} & \begin{array}{c}\text { Superoxide } \\ \text { Radical } \\ \text { (Triplet) }\end{array} & \begin{array}{c}\text { Peroxide } \\ \left(\mathrm{H}_{2} \mathrm{O}_{2}\right)\end{array} & \begin{array}{c}\text { Hydroxyl } \\ \text { radical }\end{array} \\ & \left(\mathrm{HO}_{2}\right) & & \end{array}$

Figure 1. The active oxygen system. Molecular oxygen is reduced to water in four single-electron steps. Reduction of non-radical forms of oxygen is a "forbidden" process and thus usually involves spin-orbit coupling by a heavy metal or a halide or excitation to singlet state. An example is Fenton's reaction, the reduction of peroxide to water and hydroxyl radical by ferrous iron. Hydroxyl radical is one of the most powerful oxidizing agents known.

\section{Oxidative damage to lipids (Lipid peroxidation)}

The peroxidation of lipids is basically damaging because the formation of lipid peroxidation products leads to spread of free radicals reactions. The important role of lipids in cellular components emphasizes the significance of understanding the mechanisms and consequences of lipid peroxidation in biological systems. Polyunsaturated fatty acids (PUFAs) serve as excellent substrates for lipid peroxidation because of the presence of active bis-allylic methylene groups. The carbon-hydrogen bonds on these activated methylene units have lower bond dissociation energies, making these hydrogen atoms more easily abstracted in radical reactions (Davies et al., 1981). The susceptibility of a particular PUFA toward peroxidation increases with an increase in the number of unsaturated sites in the lipid chain (Nagaoka et al., 1990).

Lipid hydroperoxides are non-radical intermediates derived from unsaturated fatty acids, phospholipids, glycolipids, cholesterol esters and cholesterol itself. Their formation occur in enzymatic or non-enzymatic reactions involving activated chemical species known as "reactive oxygen species" (ROS) which are responsible for toxic effects in the body via various tissue damages. These ROS include among others hydroxyl radicals, lipid oxyl or peroxyl radicals, singlet oxygen, and peroxinitrite formed from nitrogen oxide (NO), all these groups of atoms behave as a unit and are now named "free radical". These chemical forms are defined as any species capable of independent existence that contains one or more unpaired electrons (those which occupy an atomic or molecular orbital by themselves). They 
are formed either by the loss of a single electron from a non-radical or by the gain of a single electron by a non-radical. They can easily be formed when a covalent bond is broken if one electron from each of the pair shared remains with each atom, this mechanism is known as homolytic fission. In water, this process generates the most reactive species, hydroxyl radical $\mathrm{OH}$.. Chemists know well that combustion which is able at high temperature to rupture $\mathrm{C}-\mathrm{C}, \mathrm{C}-\mathrm{H}$ or $\mathrm{C}-\mathrm{O}$ bonds is a free-radical process. The opposite of this mechanism is the heterolytic fission in which, after a covalent break, one atom receives both electrons (this gives a negative charge) while the other remains with a positive charge.Lipid peroxidation leads to the breakdown of lipids and to the formation of a wide array of primary oxidation products such as conju- dienes or lipid hydroperoxides, and secondary products including MDA, F2-isoprostane or expired pentane, ethane or hexane. Measurement of conjugated dienes is interesting because it detects molecular reorganisation of poly- unsaturated fatty acids during the initial phase lipid peroxidation. Lipid hydroperoxide is another marker of the initial.(reaction of FR and is a specific marker of cellular damage. Other products are often used to measure oxida- stress but have the disadvantage of being secon- dary oxidation products. One of them, MDA, is during fatty acid auto-oxidation. This sub- is most commonly measured by its reaction with thiobarbituric acid, which generates thiobarbi- turic acid reactive substances (TBARS). Although of the results.MDA overestimation), this method is accepted as a the general marker of lipid peroxidation but results are subject to caution (Sies, 1997). In addition, some studcable ies tend to show that MDA is not an adapted method ucts used for such methods.

The peroxidation of lipids involves three distinct steps: initiation, propagation and termination. The initiation phase of lipid peroxidation may proceed by the reaction of an activated oxygen species such as singlet oxygen (1O2), O2-, or $\mathrm{HO} \cdot$ with a lipid substrate or by the breakdown of preexisting lipid hydroperoxides by transition metals. In the former case, peroxidation occurs by abstraction of a hydrogen atom from a methylene carbon in the lipid substrate $(\mathrm{LH})$ to generate a highly reactive carbon-centered lipid radical (L·) (Kelly et al., 1998). In the propagation phase of lipid peroxidation, molecular oxygen adds rapidly to $\mathrm{L}$ at a diffusion controlled rate to produce the lipid peroxyl radical (LOO·). The peroxyl radical can abstract a hydrogen atom from a number of in vivo sources, such as DNA and proteins, to form the primary oxidation product, a lipid hydroperoxide (LOOH). Alternatively, antioxidants such as $\alpha$-tocopherol $(\alpha-\mathrm{TOH})$ can act as excellent hydrogen atom donors, generating $\mathrm{LOOH}$ and the relatively inert $\alpha$-tocopherol phenoxyl radical $(\alpha-$ TO·). In the absence of antioxidants or other inhibitors, LOO can abstract a hydrogen from another lipid molecule (LH), producing another highly reactive carbon centered radical $(\mathrm{L} \cdot)$, which then propagates the radical chain as presented in Figure 2 (Waldeck and Stocke, 1996). The lipid hydroperoxide $(\mathrm{ROOH})$ is unstable in the presence of iron or other metal catalysts because $\mathrm{ROOH}$ will participate in a Fenton reaction leading to the formation of reactive alkoxy radicals. Therefore, in the presence of irron, the chain reactions are not only propagated but amplified. Among the degradation products of $\mathrm{ROOH}$ are aldehydes, such as malondialdehyde, and hydrocarbons, such as ethane and ethylene, which are commonly measured end products of lipid peroxidation (Sener et al., 2004). 


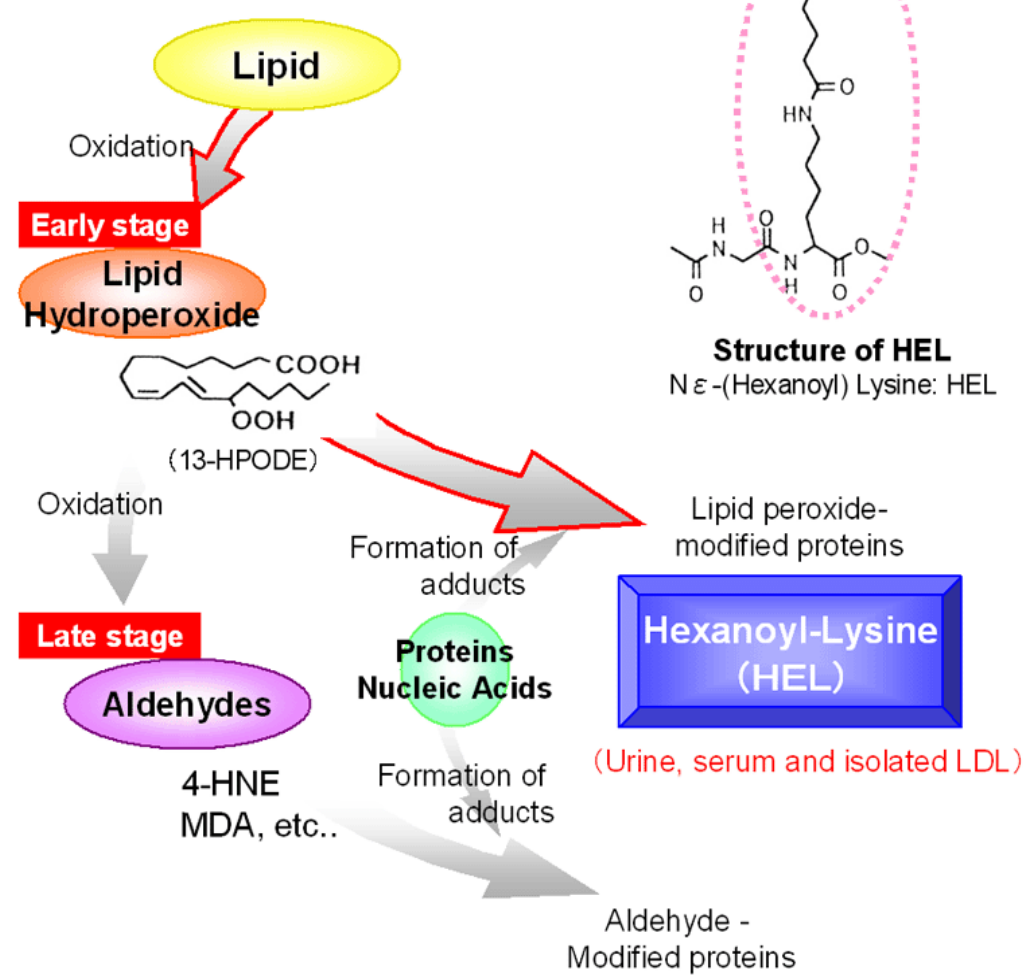

Abbreviations: NRP, nonradical product; LOOH, lipid hydroperoxide; $\alpha$-TOH, $\alpha$-tocopherol; $\alpha$-TO, $\alpha$-TOH radical; LH, lipid substrate; LOO, lipid peroxyl radical. Adapted from Waldeck and Stocke(1996)

Figure 2. Overview of lipid peroxidation.

During peroxidation pathway via reactive intermediates, several end products are formed such as aldehyde [malondialdehyde + 4-hydroxynonenal], pentane and ethane, 2,3 transconjugated diens, isoprostains and chlesteroloxides. The biological activities of MDA and other aldehydes include cross-linking with DNA and proteins, which alters the function/activity of these molecules. MDA $+4 \mathrm{HNE}$ have shown tissue toxicity. MDA can react with amino and thiol groups, the aldehydes are more diffusible than free radicals, which means damage is exported to distance sites. Aldehydes are quickly removed from cells as several enzymes control their metabolism (Ustinova and Riabinin 2003).

\section{Antioxidants}

To minimize the negative effects of ROS generated by any pro-oxidant, endogenous defensive mechanisms called antioxidant defense (AD) system, which utilizes enzymatic and non-enzymatic mechanisms. Antioxidants are naturally occurring substances that 
combat oxidative damage in biological entities. An antioxidant achieves this by slowing or preventing the oxidation process that can damage cells in the body. This it does by getting oxidized itself in place of the cells. Thus an antioxidant can also be termed as a reducing agent. Antioxidants are considered as important in the fight against the damage that can be done by free radicals produced due to oxidative stress. Although the human body has its own defenses against oxidative stress, these become weak with age or in the case of an illness. Although, antioxidants are sold in various forms as dietary supplements there is no clinching clinical evidence in favor of antioxidants as beneficial in maintaining health and preventing disease. However, there is a lot of anecdotal evidence that those who partake of antioxidant-rich food are better protected against problems such as heart disease, macular degeneration, diabetes, and cancer. Antioxidants are either hydrophilic or hydrophobic. Water soluble or hydrophilic antioxidants are active in the blood plasma while the water insoluble antioxidants protect the cell membranes. How do antioxidants work? Antioxidants work by bringing under control the rogue and unstable oxygen molecules that have an odd number of electrons. These oxygen molecules known as free radicals are highly reactive; they attack cells, DNA, and protein thereby accelerating the aging process. The antioxidants work in harmony and the efficacy of one antioxidant depends upon the availability and concentration of another. Essentially, antioxidants work by donating an electron to the unstable free radical. This stabilizes the free radical and converts it into a harmless compound that may safely be removed from the body. Antioxidants are segregated into two classes based on their mode of operating. They can either be chainbreaking or preventive. Chain-breaking antioxidants such as vitamins $\mathrm{E}$ and $\mathrm{C}$ halt the process of radical formation by stabilizing free radical molecules so that the chain-like process of radical formation is arrested. Preventive antioxidants such as superoxide dismutase and catalase prevent chain initiations by scavenging for initiator radicals and stabilizing them. They also stabilize transition metal radicals like iron and copper. These metals work as catalysts in the production of free radicals. Antioxidants and their various forms. Antioxidants are chiefly available to us through vitamins, enzymes, and minerals. Vitamin E is actually a group of eight tocopherols. Alpha-tocopherol is the most widely available tocopherol and also the most potent in terms of its effect on the body. Vitamin $\mathrm{E}$ is fat-soluble and protects cell membranes that are mainly composed of fatty acids. Vitamin $\mathrm{C}$ or ascorbic acid is water-soluble and it scavenges for free radicals that are present in aqueous environments within the human body. Beta carotene is also water soluble and is particularly effective in tackling free radicals in areas of low oxygen concentration. Selenium, manganese and zinc are trace elements that are important components of several antioxidant enzymes such as superoxide dismutase (SOD), catalase (CAT) and glutathione peroxidase. Enzymes work as both primary and secondary antioxidants and help repair oxidized DNA and target lipids that are oxidized. Other substances that are now being considered for their antioxidant properties include uric acid and phytochemicals found in plants.

Fruits and vegetables that have been identified as sources of powerful antioxidants help people counter the risk of heart ailments and different types of cancers. However, there is 
a possibility that these benefits obtained from fruits and vegetables could be a result of not just antioxidants but a mix that includes flavonoids as well. Although, clinical trials have not put forth conclusive evidence in favor of antioxidants as being helpful to our health the vast number of observational studies and anecdotal evidence offers a very strong suggestion that antioxidants are indeed of much use in keeping the body healthy. It is only a matter of time before scientists unravel the exact mechanism that governs the working of antioxidants in the body. Most nutritionists agree that the best source of antioxidants is natural food. One should try and avoid supplements if possible. It is also important to keep in mind that a high dosage of antioxidant supplements can have a detrimental effect on the body. Excessive vitamin $\mathrm{E}$ can lead to blood hemorrhage. Vitamin $C$ in large amounts can cause diarrhea and also atherosclerosis. High amounts of selenium can cause hair loss and rashes on the skin. Antioxidants are thought to protect the body against the destructive effects of free radicals. Antioxidants neutralize free radicals by donating one of their own electrons, ending the electron- gain reaction. The antioxidant nutrients themselves don't become free radicals by donating an electron because they are stable in either form. They act as scavengers, helping to prevent cell and tissue damage that could lead to cellular damage and disease (Reiter, 2003; Reiter et al., 2004).

A rangeor of antioxidants are active in the body including enzymatic and non-enzymatic. All of them can to redox status. Redox status is directly linked and be intracellular or extracellular antioxidants. The body produces several enzymes, including superoxide dismutase (SOD), catalase (CAT), glutathione peroxidase (GSHPX), and glutathione reductase (GR) that neutralize many types of free radicals. Supplements of these enzymes are available for oral administration. However, their absorption is probably minimal at best. Supplementing with the "building blocks" the body requires to make SOD, catalase, and glutathione peroxidase may be more effective. These building block nutrients include the minerals manganese, zinc, and copper for SOD and selenium for GSHPX. While the nonenzymatic defense consists of substances of low molecular weight such as reduced glutathione vitamin C, vitamin E, beta-carotene, lutein, lycopene, vitamin B2, coenzyme Q10, and cysteine (an amino acid). Herbs, such as bilberry, turmeric (curcumin), grape seed or pine bark extracts, and ginkgo can also provide powerful antioxidant protection for the body. Melatonin is a hormone secreted by pineal gland and proves to be powerful antioxidant and free radical scavenger (Yang et al.,2002 and Koc et al., 2003).

\subsection{Enzymatic antioxidants}

Antioxidant enzymes include superoxide dismutase (SOD), catalase (CAT), glutathione peroxidase (GSHPX), and glutathione reductase (GR). Non-en-zymatic antioxidants include a variety of FR quenchers such as vitamin A (retinol), vitamin C - (ascorbic acid), vitamin E (tocopherol), flavonoids, thiols (including glutathione [GSH], ubidecarenone uric acid, bilirubin, ferritin) and micronutrients (iron, copper, zinc, selenium, mangawhich which act as enzymatic cofactors. The antioxidant system efficiency depends on nutritional ineccentric 
(vitamins and micronutrients) and on endoge nous antioxidant enzyme production, which can be modified by exercise, training, nutrition and agdative Moreover, the antioxidant system efficiency is important in sport physiology because exercise increases the production of FR.

\subsubsection{Superoxide dismutase (SOD, EC 1.15.1.1)}

Superoxide dismutase (SOD) is the major defence upon superoxide radicals and is the first defence line against oxidative stress. SOD represents a group of enzymes that catalyse the dismutation of $\mathrm{O} 2-$ and the formation of H2O2. SOD is an enzyme (EC 1.15.1.1) discovered by McCord and Fridovich, which plays an important role in the defense mechanism of biological cells exposed to oxygen (McCord and Fridovich 1969). SOD catalyzes the dismutation of superoxide anion radical (O 2 - -) into an oxygen molecule and a hydrogen peroxide. This reaction is recognized as an antioxidant system that protects cells from superoxide toxicity. There are several types of SOD, depending on the type of metal ion. Three major isoforms of mammalian SOD have been identif ed with different tissue distributions (Zelko 2002). Cu/Zn - SOD (SOD1) exists in the cytoplasm, lysosomes, and nuclear compartments of mammalian cells (Bannister and et al., 1987; Zelko et al., 2002). In humans, the liver has a relatively high amount and activity of SOD1 (Nozik-Grayck et al., 2005). Human SOD1 is a homodimer containing one copper ion and one zinc ion in each 16 - kDa subunit which consists of 153 amino acids. The copper ion is held by interaction with imidazolate ligands of the histidine residues in SOD1 in the enzymatic active site. The zinc ion ( $\mathrm{Zn} 2+$ ) contributes to the stabilization of the enzyme (Johnson and Giulivi , 2005).

\subsubsection{Catalase (CAT, EC 1.11.1.6)}

Catalse (CAT) is one of the major antioxidant enzymes (Scandalios et al., 1997). It is one of the first enzymes to be purified and crystallized and has gained a lot of attention in recent years because of its link to cancer, diabetes and aging in humans and animals (Preston et al., 2001). It is present in every cell and in particular in cell structures that use oxygen in order to detoxify toxic substances and produce $\mathrm{H} 2 \mathrm{O} 2$. Catalase converts $\mathrm{H} 2 \mathrm{O} 2$ into water andoxygen (Greenwald, 1990 ; Yasminch and Theologides, 1993). Catalase can also use H2O2 in order to detoxify some toxic substances via a peroxidase reaction (Mayo et al., 2003). There are many evidences that the changes of catalase activity as well as the mechanisms of its regulation are essential in the response to stress situations which catalyzes the dismutation of $\mathrm{H} 2 \mathrm{O} 2$, forming $\mathrm{O} 2$ and $\mathrm{H} 2 \mathrm{O}$ resulting good protection the cells from the toxic effects of hydrogen peroxide (Brioukhanov and Netrusor, 2004).

\subsubsection{Glutathione peroxidase (GPX, EC 1.11.1.9)}

GPX was discovered in 1957 ny Mills. It exists in cell cytosol and mitochondria and has the ability to transform $\mathrm{H} 2 \mathrm{O} 2$ into waterThis reaction uses GSH and transforms it into oxidised glutathione (GSSG). GPX and CAT have the same action upon $\mathrm{H} 2 \mathrm{O} 2$, debut GPX is more 
efficient with high ROS concentra-tion and CAT has an important action with lower $\mathrm{H} 2 \mathrm{O} 2$ concentration. GPx is a glycoprotein containing a single selenocysteine residue at the active center of each subunit. To protect biological organisms from oxidative damage, GPx catalyses the reduction of hydrogen peroxide and lipid hydroperoxides to water and their corresponding alcohols, respectively, as follows (Antunes et al., 2002): $\mathrm{ROOH}+2 \mathrm{GSH} \rightarrow \mathrm{ROH}$ $+\mathrm{GSSG}+\mathrm{H} 2 \mathrm{O} 2$ where reduced monomeric glutathione (GSH) is essential as a hydrogen donor, and GSH is oxidized to glutathione disulf de (GSSG). There are five main mammalian isozymes, which vary in the structure (amino acid sequence and subunit), tissue distribution (liver, kidney,erythrocyte, blood plasma, among others), location (cytoplasm, intestine, extracellular f uid), and substrate specif city (hydrogen peroxide and lipid hydroperoxides) (Dudek et al., 2002). GXP is a selenium dependent enzyme that is ubiquitously expressed and protects cells against oxidative damage by reducing hydrogen peroxide and a wide range of organic peroxides with reducing glutathione (Arthur, 2000). It has been suggested that GPX has anti - inflammatory activity in the cardiovascular system. An increase in cytosolic GPx is linked to a lower risk of cardiovascular disease (Blankenberg et al. 2003).

\subsubsection{Glutathione reductase (GR, EC 1.6.4.2)}

Glutathione Reductase (GR) is a key enzyme of glutathione metabolism and is widespread in all tissues and blood cells. It a flavin enzyme involved in the defense of the erythrocyte against hemolysis. This enzyme catalyses reduction of oxidized glutathione (GSSG) to reduced glutathione (GSH) in the presence of NADPH and maintains a high intracellular GSH/GSSG ratio of about 500 in red blood cells (Kondo et al., 1980). GR is important not only for the maintaining thr required GSH level but also for reducing protein thiols to their native state.This enzyme is conserved between all kingdoms. In bacteria, yeasts and animals, one GR gene is found, however in plant genomes two GR genes are enclosed. Under normal conditions, GSH and GR are involved in the detoxification of $\mathrm{H} 2 \mathrm{O} 2$ generated in the light by the Mehler reaction in chloroplasts. Disturbances GH level have been correlated with oxidative stress induced by various factors including toxicity, pollutants, inflammation and different diseases particularly red blood cell defects.

\subsubsection{Glutathione-S-transferase (GST, EC 2.5.1.18)}

Glutathione-S-Transferase (GST) catalyzes the conjugation with glutathione of a number of electrophilic xenobiotics, including several carcinogens, mutagens and anticancer drugs (Hayes and Pulford, 1995). These electrophiles are made less reactive by conjugation with glutathione and the conjugates are thought to be less toxic to the cell. Consiquently, GSTs are believed to play an important role in the defense of cells against these zenobiotic toxins. Several antineoplastic drugs particularly the reactive electrophilic alkylating agents, can form conjugates with glutathione both spontaneously and in GST-catalyzed reactions (Awasthi et al., 1996). Morever, Some studies reported that there are a good association between cellular resistance to some anticancers drugs and expression of particular isozymes of GST (Hayes and Pulford, 1995). 


\subsubsection{Glutathione (GSH)}

Gultathione (GSH) is a small molecule found in almost every cell (Anderson, 1997). It is the smallest intrecellular thiol ( $\mathrm{SH}$ ) molecule. Its high electron-donating capacity (high negative redox potential) combined with high intracellular concentration generate great reducing power (Kidd, 1997). Glutathione is a cysteine-containing peptide found in most forms of aerobic life. It is not required in the diet and is instead synthesized in cells from its constituent amino acids (Meister and Anderson, 1983; Meister, 1988). It can react non-enzymatically with ROS and GSH peroxidase catalyses the destruction of hydrogen peroxide and hydroperoxides resulting in its oxidation to the disulphide form (GSSG). Firstly, glutathione is the major antioxidant produced by the cell protecting it from free radicals as oxygen radicals which are highly reactive substances can damage or destroy key cell components. Its antioxidant properties result from the thiol group in its cysteine moiety is a reducing agent and can be reversibly oxidized and reduced. In cells, glutathione is maintained in the reduced form by the enzyme glutathione reductase and in turn reduces other metabolites and enzyme systems, such as ascorbate in the glutathione-ascorbate cycle, glutathione peroxidases and glutaredoxins, as well as reacting directly with oxidants. Due to its high concentration and its central role in maintaining the cell's redox state, glutathione is one of the most important cellular antioxidants. In some organisms glutathione is replaced by other thiols, such as by mycothiol in the Actinomycetes, bacillithiol in some Gram-positive bacteria, or by trypanothione in the Kinetoplastids (Meister and Larsson, 1995). Secondly, GSH is a very important detoxifing agent, enabling the body to get rid of undesirable toxins and pollutants. It forms a soluble compound with the toxin that can then be excreted through the urine or the gut. The liver and kidneys contain high levels of GSH as they have the greatest exposure to toxins. The lung are also rich in glutathione partly for the same reason. Thirdly, GSH plays a crucial role in maintaining a normal balance between oxidation and anti-oxidation. This in turn regulates many of the cell's vital functions such as the synthesis and repair of DNA, the synthesis of proteins and the activation, maintaining the essential thiol status of protein, immune function, regulate nitric oxide homeostasis, modulate the activity of neurotransmitter receptors and regulation of enzymes (Oja et al., 2000; Hogg, 2002). The lower level of GSH is related to different physiological and biochemical disturbances.

\subsubsection{Ascorbic acid}

Ascorbic acid or "vitamin C" is a monosaccharide oxidation-reduction (redox) catalyst found in both animals and plants (Peake, 2003). As one of the enzymes needed to make ascorbic acid has been lost by mutation during primate evolution, humans must obtain it from the diet; it is therefore a vitamin. Most other animals are able to produce this compound in their bodies and do not require it in their diets. Ascorbic acid is required for the conversion of the procollagen to collagen by oxidizing proline residues to hydroxyproline. In other cells, it is maintained in its reduced form by reaction with glutathione, which can be catalysed by protein disulfide isomerase and glutaredoxins. Ascorbic acid is redox catalyst which can reduce, and thereby neutralize, reactive oxygen species such as hydrogen peroxide. In 
addition to its direct antioxidant effects, ascorbic acid is also a substrate for the redox enzyme ascorbate peroxidase, a function that is particularly important in stress resistance in plants. Ascorbic acid is present at high levels in all parts of plants and can reach concentrations of 20 millimolar in chloroplasts. It act as a marked antioxidant that help in the treatement of different diseases such as cancer and cardiovascular (Coulter et al., 2006 ; Cook et al., 2007).

\subsubsection{Melatonin}

Melatonin (N-acetyl-5-methoxytryptamine), is synthesized from serotonin in the pineal gland which contains all the enzymes necessary for the methoxylation and acetylation reactions. Melatonin is released in mammals during the dark-phase of the circadian cycle, and declines with age (Tan et al., 2001). It is able to reduce the free radical formation which follows the interaction between transition metal ions and amyliod-beta peptide (Zatta et al., 2003). As a free radical scavenger melatonin exhibits several important properties: It has both lipophilic and hydrophilic and it passes all bio-barriers, e.g. blood brain barrier and placenta (Wakatsuki et al., 1999).

Reiter (1995) reported that melatonin seems to be more effective than other antioxidants (e.g. mannitol, glutathione and vitamin E) in protecting against oxidative damage. Thus, it may provide protection against diseases that cause degenerative or proliferative changes by shielding macromolecules, particularly DNA from such injuries. Besides its direct free radical scavenging action, melatonin functions as an indirect antioxidant by stimulating the activities of antioxidiative enzymes in addition to protecting against lipid peroxidation (Undeger et al., 2004).

Melatonin has been found to be a direct free radical scavenger and an indirect antioxidant that, may have an active role in protection against genetic damage due to endogenously produced free radicals and it may be of use in reducing damage from physical and chemical mutagens and carcinogens that generate free radicals (Bandyopadhyay et al., 2000).

\subsubsection{Tocopherols and tocotrienols (vitamin E)}

Vitamin $\mathrm{E}$ is the collective name for a set of eight related tocopherols and tocotrienols, which are fat-soluble vitamins with antioxidant properties (Roberts et al., 2007). Of these, $\alpha$ tocopherol has been most studied as it has the highest bioavailability, with the body preferentially absorbing and metabolising this form. It has been claimed that the $\alpha$ tocopherol form is the most important lipid-soluble antioxidant, and that it protects membranes from oxidation by reacting with lipid radicals produced in the lipid peroxidation chain reaction. This removes the free radical intermediates and prevents the propagation reaction from continuing (Cook et al., 2007). This reaction produces oxidised $\alpha$ tocopheroxyl radicals that can be recycled back to the active reduced form through reduction by other antioxidants, such as ascorbate, retinol or ubiquinol.This is in line with findings showing that $\alpha$-tocopherol, but not water-soluble antioxidants, efficiently protects 
glutathione peroxidase 4 (GPX4)-deficient cells from cell death. GPx4 is the only known enzyme that efficiently reduces lipid-hydroperoxides within biological membranes. However, the roles and importance of the various forms of vitamin $\mathrm{E}$ are presently unclear, and it has even been suggested that the most important function of $\alpha$-tocopherol is as a signaling molecule, with this molecule having no significant role in antioxidant metabolism. The functions of the other forms of vitamin E are even less well-understood, although $\gamma$ tocopherol is a nucleophile that may react with electrophilic mutagens, and tocotrienols may be important in protecting neurons from damage. However it has a protective action against different diseases including cancer (Coulter et al., 2006).

\subsection{Total antioxidant capacity}

Epidemiologic studies have demonstrated an inverse association between consumption of fruits and vegetables and morbidity and mortality from degenerative diseases. The antioxidant content of fruits and vegetables may contribute to the protection they offer from disease. Because plant foods contain many different classes and types of antioxidants, knowledge of their total antioxidant capacity (TAC), which is the cumulative capacity of food components to scavenge free radicals, would be useful for epidemiologic purposes. To accomplish this, a variety of foods commonly consumed in Italy, including 34 vegetables, 30 fruits, 34 beverages and 6 vegetable oils, were analyzed using three different assays, i.e., Trolox equivalent antioxidant capacity (TEAC), total radical-trapping antioxidant parameter (TRAP) and ferric reducing-antioxidant power (FRAP). These assays, based on different chemical mechanisms, were selected to take into account the wide variety and range of action of antioxidant compounds present in actual foods. Among vegetables, spinach had the highest antioxidant capacity in the TEAC and FRAP assays followed by peppers, whereas asparagus had the greatest antioxidant capacity in the TRAP assay. Among fruits, the highest antioxidant activities were found in berries (i.e., blackberry, redcurrant and raspberry) regardless of the assay used. Among beverages, coffee had the greatest TAC, regardless of the method of preparation or analysis, followed by citrus juices, which exhibited the highest value among soft beverages. Finally, of the oils, soybean oil had the highest antioxidant capacity, followed by extra virgin olive oil, whereas peanut oil was less effective. Such data, coupled with an appropriate questionnaire to estimate antioxidant intake, will allow the investigation of the relation between dietary antioxidants and oxidative stress-induced diseases (Pellegrini et al., 2003 ; Puchau et al., 2009 ; Dilis and Trichopoulou, 2010).

\section{Nutritional therapy with natural antioxidants}

Antioxidants have been the focus of research on the relationship between The role of dietary factors in protecting against the change from native to oxidized LDL has received considerable attention. An overview of epidemiological research suggests that individuals with the highest intakes of antioxidant vitamins, whether through diet or supplements, tend to lower of various disease. Research examining the effects of a diet rich in fruits and vegetables on disease has been carried out using several types of study.There is strong 
scientific evidence to support an increase in intakes of vegetables and fruit in the prevention of disease. Further research is required to clarify which particular components of fruit and vegetables are responsible for their protective effects. Numerous epidemiological studies have indicated that diets rich in fruits and vegetables are correlated with a reduced risk of chronic diseases (German, 1999; Benzie, 2003; Hassan, 2005; Hassan and Yosef, 2009; Hassan et al., 2010). It is probable that antioxidants, present in the fruits and vegetables such as polyphenols, carotenoids, and vitamin $\mathrm{C}$, prevent damage from harmful reactive oxygen species, which either are continuously produced in the body during normal cellular functioning or are derived from exogenous sources (Gate et al. 1999). The possible protective effect of antioxidants in fruits and vegetables against ROS has led people to consume antioxidant supplements against chronic diseases.

\section{Nutritional factors as natural antioxidants agents in alleviating the oxidative stress induced by environmental pollutents: Some experimental studies for the author}

\subsection{Mitigating effects of antioxidant properties of black berry juice on sodium fluoride induced hepatotoxicity and oxidative stress in rats}

Fluorosis is a serious public health problem in many parts of the world. As in the case of many chronic degenerative diseases, increased production of reactive oxygen species has been considered to play an important role, even in the pathogenesis of chronic fluoride toxicity. Black berry is closely linked to its protective properties against free radical attack. Therefore, the aim of this study was to demonstrate the role of black berry juice (BBJ) in decreasing the hepatotoxicity and oxidative stress of sodium fluoride ) NaF). Results showed that $\mathrm{NaF}$ caused elevation in liver TBARS and nitric oxide (NO), and reduction in superoxide dismutase (SOD), catalase (CAT), total antioxidant capacity (TAC) and glutathione (GSH.( Plasma transaminases (AST and ALT), creatine kinase (CK), lactate dehydrogenase (LDH), total lipids )TL), cholesterol, triglycerides (TG), and low density lipoprotein-cholesterol (LDL-c) were increased, while high density lipoprotein-cholesterol $(\mathrm{HDL}-\mathrm{c})$ was decreased. On the other hand, BBJ reduced NaF-induced TBARS, NO, TL, cholesterol, TG, LDL-C, AST, ALT, CK and LD. Moreover, it ameliorated NaFinduced decrease in SOD, CAT, GSH, TAC and HDL-c. Therefore, the present results revealed that $\mathrm{BBJ}$ has a protective effect against $\mathrm{NaF}$-induced hepatotoxicity by antagonizing the free radicals generation and enhancement of the antioxidant defence mechanisms. (Hassan and Yousef, 2009).

\subsection{Evaluation of free radical-scavenging and antioxidant properties of black berry against fluoride toxicity in rats}

Oxidative damage to cellular components such as lipids and cell membranes by free radicals and other reactive oxygen species is believed to be associated with the development of 
degenerative diseases. Fluoride intoxication is associated with oxidative stress and altered anti-oxidant defense mechanism. So the present study was extended to investigate black berry anti-oxidant capacity towards superoxide anion radicals, hydroxyl radicals and nitrite in different organs of fluoride-intoxicated rats. The data indicated that sodium fluoride $(10.3$ $\mathrm{mg} / \mathrm{kg} \mathrm{bw}$ ) administration induced oxidative stress as evidenced by elevated levels of lipid peroxidation and nitric oxide in red blood cells, kidney, testis and brain tissues. Moreover, significantly decreased glutathione level, total anti-oxidant capacity and superoxide dismutase activitywere observed in the examined tissues. On the other hand, the induced oxidative stress and the alterations in anti-oxidant system were normalized by the oral administration of black berry juice $(1.6 \mathrm{~g} / \mathrm{kg} \mathrm{bw})$. Therefore it can be concluded that black berry administration could minimize the toxic effects of fluoride indicating its free radicalscavenging and potent anti-oxidant activities. (Hassan and Fattoh, 2010)

\subsection{Garlic oil as a modulating agent for oxidative stress and neurotoxicity induced by sodium nitrite in male albino rats}

In the present study, we investigated the neurobiochemical alterations and oxidative stress induced by food preservative; sodium nitrite (NaNO2) as well as the role of the garlic oil in amelioration of the neurotoxicity in male albino rats. Serum and brain homogenates of the rats received NaNO2 $(80 \mathrm{mg} / \mathrm{kg}$ body weight) for 3 months exhibited significant decrease in acetylcholine esterase (AChE) activity as well as the levels of phospholipids, total protein and the endogenous antioxidant system (glutathione; GSH and superoxide dismutase; SOD). In contrast, lactic dehydrogenase (LDH) activity, brain thiobarbituric acid reactive substances (TBARS) and nitric oxide (NO) levels were significantly increased. On the other hand, the oral administration of garlic oil ( $5 \mathrm{ml} / \mathrm{kg}$ body weight) daily for 3 months significantly improved the neurobiochemical disorders and inhibited the oxidative stress induced by $\mathrm{NaNO} 2$ ingestion. So, this study reveals the neural toxic effects of NaNO2 by exerting oxidative stress and retrograde the endogenous antioxidant system. However, garlic oil has a promising role in attenuating the obtained hazard effects of sodium nitrite by its high antioxidant properties which may eventually be related with the preservation of SOD activity and primary mitochondrial role against nitrite-induced neurotoxicity in rats . (Hassan et al., 2010).

\subsection{In vivo evidence of hepato-and-reno-protective effect of garlic oil against sodium nitrite-induced oxidative stress}

Sodium nitrite (NaNO2), a food color fixative and preservative, contributes to carcinogenesis. We investigated the protective role of garlic oil against NaNO2-induced abnormalities in metabolic biochemical parameters and oxidative status in male albino rats. NaNO2 treatment for a period of three months induced a significant increase in serum levels of glucose, aspartate aminotransferase (AST), alanine aminotransferase (ALT), alkaline phosphatase (ALP), bilirubin, urea and creatinine as well as hepatic AST and ALT. 
However, significant decrease was recorded in liver ALP activity, glycogen content, and renal urea and creatinine levels. In parallel, a significant increase in lipid peroxidation, and a decrease in glutathione content and catalase activity were observed in the liver and the kidney. However, garlic oil supplementation showed a remarkable amelioration of these abnormalities. Our data indicate that garlic is a phytoantioxidant with powerful chemopreventive properties against chemically-induced oxidative stress (Hassan et al., 2009).

\subsection{Ameliorating effect of chicory (Cichorium intybus L)-supplemented diet against nitrosamine precursors-induced liver injury and oxidative stress in male rats}

The current study was carried out to elucidate the modulating effect of chicory (Cichorium intybus L.)-supplemente diet against nitrosamnine-induced oxidative stress and hepatotoxicity in male rats. Rats were divided into four groups and treated for 8 weeks as follow: group 1 served as control; group 2 fed on chicory-supplemented diet $(10 \% \mathrm{w} / \mathrm{w})$; group 3 received simultaneously nitrosamine precursors [sodium nitrite $(0.05 \%$ in drinking water) plus chlorpromazine $(1.7 \mathrm{mg} / \mathrm{kg}$ body weight)] and group 4 received nitrosamine precursors and fed on chicory-supplemented diet. The obtained results revealed that rats received nitrosamine precursors showed a significant increase in liver TBARS and total lipids, total cholesterol, bilirubin, and enzymes activity (AST, ALT, ALP and GGT) in both serum and liver. While a significant decrease in the levels of GSH, GSH-Rx, SOD, catalase, total protein and albumin was recorded. On the other hand, chicory-supplemented diet succeeded to modulate these observed abnormalities resulting from nitrosamine compounds as indicated by the reduction of TBARS and the pronounced improvement of the investigated biochemical and antioxidant parameters. So, it could be concluded that chicory has a promising role and it worth to be considered as a natural substance for ameliorating the oxidative stress and hepatic injury induced by nitrosamine compounds (Hassan and Yousef, 2010).

\section{Summary}

\subsection{Oxidative stress}

The term oxidative stress refers to a condition in which cells are subjected to excessive levels of molecular oxygen or its chemical derivatives called reactive oxygen species (ROS).Under physiological conditions, the molecular oxygen undergoes a series of reactions that ultimately lead to the generation of superoxide anion $\left(\mathrm{O}_{2}-\right)$, hydrogen peroxide $\left(\mathrm{H}_{2} \mathrm{O}_{2}\right)$ and $\mathrm{H}_{2} \mathrm{O}$. Peroxynitrite (OONO-), hypochlorus acid $(\mathrm{HOCl})$, the hydroxyl radical $(\mathrm{OH}$.), reactive aldehydes, lipid peroxides and nitrogen oxides are considered among the other oxidants that have relevance to vascular biology.

Oxygen is the primary oxidant in metabolic reactions designed to obtain energy from the oxidation of a variety of organic molecules. Oxidative stress results from the metabolic 
reactions that use oxygen, and it has been defined as a disturbance in the equilibrium status of pro-oxidant/anti-oxidant systems in intact cells. This definition of oxidative stress implies that cells have intact pro-oxidant/anti-oxidant systems that continuously generate and detoxify oxidants during normal aerobic metabolism. When additional oxidative events occur, the pro-oxidant systems outbalance the anti-oxidant, potentially producing oxidative damage to lipids, proteins, carbohydrates, and nucleic acids, ultimately leading to cell death in severe oxidative stress. Mild, chronic oxidative stress may alter the anti-oxidant systems by inducing or repressing proteins that participate in these systems, and by depleting cellular stores of anti-oxidant materials such as glutathione and vitamin E (Laval, 1996). Free radicals and other reactive species are thought to play an important role oxidative stress resulting in many human diseases. Establishing their precise role requires the ability to measure them and the oxidative damage that they cause (Halliwell and Whiteman, 2004).

A basic approach to study oxidative stress would be to measure some products such as (i) free radicals; (ii) radical-mediated damages on lipids, proteins or DNA molecules; and iii) antioxidant enzymatic activity or concentration.

\subsection{Free radicals}

Free radicals are reactive compounds that are naturally produced in the human body. They can exert positive effects (e.g. on the immune system) or negative effects (e.g. lipids, proteins or DNA oxidation). Free radicals are normally present in the body in minute concentrations. Biochemical processes naturally lead to the formation of free radicals, and under normal circumstances the body can keep them in check. If there is excessive free radical formation, however, damage to cells and tissue can occur (Wilson, 1997). Free radicals are toxic molecules, may be derived from oxygen, which are persistently produced and incessantly attack and damage molecules within cells; most frequently, this damage is measured as peroxidized lipid products, protein carbonyl, and DNA breakage or fragmentation. Collectively, the process of free radical damage to molecules is referred to as oxidative stress (Reiter et al., 1997).To limit these harmful effects, an organism requires complex protection the antioxidant system. This system consists of antioxidant enzymes (catalase, glutathione peroxidase, superoxide dismutase) and non-enzymatic antioxidants (e.g. vitamin E [tocopherol], vitamin A [retinol], vitamin C [ascorbic acid], glutathione and uric acid). An imbalance between free radical production and antioxidant defence leads to an oxidative stress state, which may be involved in aging processes and even in some pathology (e.g. cancer and Parkinson's disease).

\subsection{Oxidative damage to lipids (Lipid peroxidation)}

The peroxidation of lipids involves three distinct steps: initiation, propagation and termination. The initiation reaction between an unsaturated fatty acid and the hydroxyl radical involves the abstraction of a $\mathrm{H}$ atom from the methylvinyl group on the fatty acid. The remaining carbon-centred radical, forms a resonance structure sharing this unpaired electron among carbons 9 to 13. In the propagation reactions, this resonance structure reacts 
with triplet oxygen, which is a biradical having two unpaired electrons and therefore reacts readily with other radicals. This reaction forms a peroxy radical. The peroxy radical then abstracts a $\mathrm{H}$ atom from a second fatty acid forming a lipid hydroperoxide and leaving another carbon centered free radical that can participate in a second $\mathrm{H}$ abstraction. Therefore, once one hydroxyl radical initiates the peroxidation reaction by abstracting a single $\mathrm{H}$ atom, it creates a carbon radical product that is capable of reacting with ground state oxygen in a chain reaction. The role of the hydroxyl radical is analogous to a "spark" that starts a fire. The basis for the hydroxyl radical's extreme reactivity in lipid systems is that at very low concentrations it initiates a chain reaction involving triplet oxygen, the most abundant form of oxygen in the cell (Benderitter et al., 2003).

The lipid hydroperoxide $(\mathrm{ROOH})$ is unstable in the presence of irron or other metal catalysts because $\mathrm{ROOH}$ will participate in a Fenton reaction leading to the formation of reactive alkoxy radicals. Therefore, in the presence of irron, the chain reactions are not only propagated but amplified. Among the degradation products of $\mathrm{ROOH}$ are aldehydes, such as malondialdehyde, and hydrocarbons, such as ethane and ethylene, which are commonly measured end products of lipid peroxidation (Sener et al., 2004). During peroxidation pathway via reactive intermediates, several end products are formed such as aldehyde [malondialdehyde + 4-hydroxynonenal], pentane and ethane, 2,3 transconjugated diens, isoprostains and chlesteroloxides. The biological activities of MDA and other aldehydes include cross-linking with DNA and proteins, which alters the function/activity of these molecules. MDA + 4HNE have shown tissue toxicity. MDA can react with amino and thiol groups, the aldehydes are more diffusible than free radicals, which means damage is exported to distance sites. Aldehydes are quickly removed from cells as several enzymes control their metabolism (Ustinova and Riabinin 2003).

\subsection{Antioxidants}

Antioxidants are thought to protect the body against the destructive effects of free radicals. Antioxidants neutralize free radicals by donating one of their own electrons, ending the electron- gain reaction. The antioxidant nutrients themselves don't become free radicals by donating an electron because they are stable in either form. They act as scavengers, helping to prevent cell and tissue damage that could lead to cellular damage and disease (Reiter, 2003).

The body produces several enzymes, including superoxide dismutase (SOD), catalase (CAT), and glutathione peroxidase (GSHPX), that neutralize many types of free radicals. Supplements of these enzymes are available for oral administration. However, their absorption is probably minimal at best. Supplementing with the "building blocks" the body requires to make SOD, catalase, and glutathione peroxidase may be more effective. These building block nutrients include the minerals manganese, zinc, and copper for SOD and selenium for GSHPX.

In addition to enzymes, many vitamins, minerals and hormones act as antioxidants in their own right, such as vitamin C, vitamin E, beta-carotene, lutein, lycopene, vitamin B2, coenzyme Q10, and cysteine (an amino acid). Herbs, such as bilberry, turmeric (curcumin), 
grape seed or pine bark extracts, and ginkgo can also provide powerful antioxidant protection for the body. Melatonin is a hormone secreted by pineal gland and proves to be powerful antioxidant and free radical scavenger (Yang et al.,2002 and Koc et al., 2003).

\subsection{Nutritional therapy with natural antioxidants}

Antioxidants have been the focus of research on the relationship between The role of dietary factors in protecting against the change from native to oxidized LDL has received considerable attention. An overview of epidemiological research suggests that individuals with the highest intakes of antioxidant vitamins, whether through diet or supplements, tend to experience $20-40 \%$ lower risk of coronary heart disease (CHD) than those with the lowest intake or blood levels (diet and disease. Research examining the effects of a diet rich in fruits and vegetables on disease has been carried out using several types of study.

There is strong scientific evidence to support an increase in intakes of vegetables and fruit in the prevention of disease. Further research is required to clarify which particular components of fruit and vegetables are responsible for their protective effects.

Numerous epidemiological studies have indicated that diets rich in fruits and vegetables are correlated with a reduced risk of chronic diseases (Banerjee and Maulik, 2002 ; Sesso et al., 2003). It is probable that antioxidants, present in the fruits and vegetables such as polyphenols, carotenoids, and vitamin $\mathrm{C}$, prevent damage from harmful reactive oxygen species, which either are continuously produced in the body during normal cellular functioning or are derived from exogenous sources (Gate et al. 1999). The possible protective effect of antioxidants in fruits and vegetables against ROS has led people to consume antioxidant supplements against chronic diseases.

\subsection{Some experimental studies for the author about food factors as a nutritional antioxidants agents in alleviating the oxidative stress induced by environmental pollutents}

1. In vivo evidence of hepato-and-reno-protective effect of garlic oil against sodium nitrite-induced oxidative stress Int. Int. J. Biol. Sci. (5)3: 249-255. (Hassan et al., 2009)

2. Mitigating effects of antioxidant properties of black berry juice on sodium fluoride induced hepatotoxicity and oxidative stress in rats. Food Chem.Toxicol., 47 2332-2337. (Hassan and Yousef, 2010).

3. Evaluation of free radical-scavenging and antioxidant properties of black berry against fluoride toxicity in rats. Food Chem.Toxicol., 48: 1999-2004. (Hassan and Fattoh, 2010).

4. Garlic oil as a modulating agent for oxidative stress and neurotoxicity induced by sodium nitrite in male albino rats. Food Chem. Toxicol., 48: 1980-1985. (Hassan et al., 2010)

5. Ameliorating effect of chicory (Cichorium intybus L)-supplemented diet against nitrosamine precursors-induced liver injury and oxidative stress in male rats. Food Chem. Toxicol., 48: 2163-2169. (Hassan and Yousef, 2010). 


\section{Author details}

Hanaa Ali Hassan Mostafa Abd El-Aal

Zoology Department, Faculty of Science, Mansoura University, Mansoura, Egypt

\section{References}

Antunes, F., Derick, H. and Cadenas, E. (2002). Relative contributions of heart mitochondria glutathione peroxidase and catalase to $\mathrm{H} 2 \mathrm{O} 2$ detoxification in in vivo conditions. Free Radic. Biol. Med., 33 (9): 1260-1267

Al-Omar, M. A., Beedham, C. and al-Sarra, I. (2004). Pathological roles of reactive oxygen species and their defence mechsnisms. Saudi Pharm. J., 12:1-18.

Arthur, J. R. (2000). The glutathione peroxidase. Cell Mol. Life Sci., 57:1825 1835.

Awasthi, S., Bajpai, K. K., Piper, J. T., Singhal, S. S., Ballaatore, A., Seifert, W. E., Awasthi. Y. C. and Ansari, G. A. S. (1996): Drug metabolism. Dispos. 24: 371-374.

Bandyopadhyay, D., Biswas, K., Bandyopadhyay, U., Reiter, R. J. and Banerjee, R. K. (2000). Melatonin protects against stress-induced gastric lesions by scavenging the hydroxyl radical. J. Pineal Res., 29: 143-151.

Banerjee, S. K. and Maulik, s. K. (2002): Effect of garlic on cardiovascular disorders. J. Nutr.,1:4-36.

Bannister, J., Bannister, W. and Rotilio, G. (1987). Aspects of the structure, function, and applications of superoxide dismutase". CRC Crit. Rev. Biochem. 22 (2): 111-180.

Benderitter, M.; Vincent-Genod, L.; Pouget, J. P. and Voisin, P. (2003): The cell membrane as a biosensor of oxidative stress induced by radiation exposure: a multiparameter investigation. Radiat. Res., 159(4): 471-483.

Benzie, I. (2003). Evolution of dietary antioxidants. Comp. Biochem. Phys., 136 (1): 113-126.

Blankenberg, S., Rupprecht, H. J., Bickel, C., Torzewski, M., Hafner, G., Tiret, L., Smieja, M., Cambien, F., Meyer, J., and Lackner, K.J. (2003). Glutathione peroxidase 1 activity and cardiovascular events in patients with coronary artery disease atherogene investigators. Engl. J. Med., 349:1605-1613

Brioukhanov, A; L; and Netrusoe, a. I. (2004): Catalase and superoxide dismutase, distribution, properties and physiological role in cells of strict anaerobes. Biochem. 69:949-962.

Ceconi, C. , Boraso, A. , Cargnoni, A. and Ferrari, R., (2003). Oxidative stress in cardiovascular disease:myth or fact? Arch. Biochem. Biophys., 420: 217 - 221 .

Ceriello, A. and Motz, E. (2004). Is oxidative stress the pathogenic mechanism underlying insulin resistance, diabetes, and cardiovascular disease? The common soil hypothesis revisited .Arterioscler. Thromb. Vasc. Biol., 24 : 816 - 823 .

Cohen, P. J. (1992): Allopurinol administered prior hepatic ischaemic in the rat prevents chemiluminescence following restoration of circulation. Can. J. Anaesth., 39: 1090-1093.

Cook, NR., Albert, CM. and Gaziano, JM. (2007). A randomized factorial trial of vitamins C and $\mathrm{E}$ and beta carotene in the secondary prevention of cardiovascular events in 
women: results from the Women's Antioxidant Cardiovascular Study. Arch. Int. Med., 167 (15): 1610-1618.

Coulter, I., Hardy, M., Morton, S., Hilton, L., Tu, W., Valentine, D. and Shekelle, P. (2006). Antioxidants vitamin $\mathrm{C}$ and vitamin e for the prevention and treatment of cancer. J. $\mathrm{f}$ general int. medici.: official j. Soc. Res. Educ. in Primary Care Int. Medici., 21 (7): 735744

Davies, AG., Griller, D., Ingold, KU., Lindsay, DA. and Walton, JC. (1981). An electron spin resonance study of pentadienyl and related radicals: homolytic fission of cyclobut-2enylmethyl radicals. J. Chem. Soc. Perkin. Trans., II:633-641.

Dherani, M. , Murthy, GV., Gupta, SK., Young, IS. and Maraini, G, Dherani, M, Murthy, GV, Gupta, SK, Young, IS, Maraini, G, Camparini, M, Price, GM, John, N, Chakravarthy, U, Fletcher, AE. (2008). Blood levels of vitamin C,carotenoids and retinol are inversely associated with cataract in a North Indian population .Invest. Ophthalmol. Vis. Sci., 49: $3328-3335$.

Dilis, V. and Trichopoulou, A. (2010). Antioxidant intakes and food sources in greek adults J. Nutr., 140: 1274-1279.

Dudek, H., Farbiszewski, R., Michno, T., Łebkowski, W. J. and Kozłowski, A. (2002). Activity of glutathione peroxidase (GSH-Px), glutathione reductase (GSSG-R) and superoxide dismutase in the brain tumors 55, 5-6: 252-256.

Fearon, IM. and Faux, SP. (2009) . Oxidative stress and cardiovascular disease: novel tools give (free)radical insight . J. Mol. Cell Cardiol., 47 : 372 - 381 .

Gate L, Paul J, Ba GN, Tew KD and Tapiero H. (1999): Oxidative stress induced in pathologies: the role of antioxidants. Biomed Pharmacother,53: 169 -180.

German, JB. (1999). Food processing and lipid oxidation". Advances in experimental medicine and biology. Advanc. Experim. Medici. Biol., 459: 23-50.

Greenwald, R. A. (1990). Superoxide dismutase and catalase as therapeutic agents for human diseases. A critical review. Free Radic. Boil. Med., 8(2): 210-219.

Halliwell, B. and Whiteman, M. (2004): Measuring reactive species and oxidative damage in vivo and in cell culture: how should you do it and what do the results mean?. Br. J. Pharmacol.., 142(2):231-255.

Hassan, H. A. (2005). The ameliorating effect of black grape juice on fluoride induced testicular toxicity in adult albino rats. Egypt. J. Zool., 44:507-525.

Hassan, H. A ; El-Agmy Sh. M; Gaur, R. L; Fernando, A. Raj, M., HG and Ouhtit A. (2009): In vivo evidence of hepato-and-reno-protective effect of garlic oil against sodium nitriteinduced oxidative stress Int. Int. J. Biol. Sci. (5)3: 249-255.

Hassan, H. A and A. M. Fattoh (2010): Evaluation of free radical-scavenging and antioxidant properties of black berry against fluoride toxicity in rats. Food Chem.Toxicol.,48:19992004.

Hassan H. A. Hafez H. S. and Zeghebar F. E. (2010): Garlic oil as a modulating agent for oxidative stress and neurotoxicity induced by sodium nitrite in male albino rats. Food Chem. Toxicol., 48: 1980-1985. 
Hassan, H. A and Yousef M. I. (2009): Mitigating effects of antioxidant properties of black berry juice on sodium fluoride induced hepatotoxicity and oxidative stress in rats. Food Chem.Toxicol., 47 2332-2337

Hassan, H. A and Yousef, M. I. (2010): Ameliorating effect of chicory (Cichorium intybus L)supplemented diet against nitrosamine precursors-induced liver injury and oxidative stress in male rats. Food Chem. Toxicol., 48: 2163-2169.

Hayes, J. D. and Pulford, D. J. (1995): The glutathione S transferase supergene family: regulation of GST and the contribution of the isoenzymes to cancer chemoprotectiion and drug resistance. Crit. Rev. Biochem. Mol. Biol., 30: 445-600.

Hogg, N. (2002): The biochemistry and physiology of S nitrosothiols. Ann. Rev. Pharmacol. Toxicol., 42:585-600.

Huang, D. Y. and Ichikawa, Y. (1994): Two different enzymes are primary responsible for retinoic acid synthesis in rabbit liver cystosol. Biochem. Biophys. Res. Commin., 205: 1278-1283.

Johnson, F. and Giulivi, C. (2005). Superoxide dismutases and their impact upon human health. Mol. Aspects Med. 26 (4-5): 340-352.

Kelly, S. A., Havrilla, C. M., Brady, T. C., Abramo, K. H. and Levin, E. D. (1998). Review of oxidative stress in toxicology: Established mammalian and emerging piscine model systems. Environ. Health Perspect., 106:375-384.

Kidd, P. M. (1997): Glutathione systemic protectant against oxidative and free radical damage. Altern. Med. Res., 1-155-176.

Koc, M.,Taysi, S.,Buyukokuroglu, M. E. and Bakan, N. (2003). The effect of melatonin against oxidative damage during total- body irradiation in rats. Radiat. Res., 160: 251-255.

Kondo, T., Dale, O.L. and Beutler, E. (1980): Glutathione transport by inside-out vesicles from human erythrocytes. Proc. Nat. Acad. Sci. Biochem., 77: 6359-6362.

Kregel, KC. and Zhang, HJ . (2007) . An integrated view of oxidative stress in aging: basic mechanisms,functional effects, and pathological considerations . Am. J. Physiol. Regul. Integr. Comp. Physiol .,292: R18 - 36 .

Laval, J. (1996): Role of DNA repair enzymes in the cellular resistance to oxidative stress. Pathol. Biol., 44: 14-24

Mayo, J. C., Tan, D. X., Sainz, R. M., Lopez-Burillo, S. and Reiter, R, J. (2003). Oxidative damage to Catalase induced by peroxyl radicals: Functional protection by melatonin and other antioxidants. Free Radic. Res., 37: 543-553.

McCord, J. M. (1985): Oxygen-derived free radicals in postischemic tissue injury. N. Eng. J. Med., 312: 159-163.

Meister, A. (1988). Glutathione metabolism and its selective modification. J. Biol. Chem., 263(33):17205-17208.

Meister, A. and Anderson, M. N. (1983). Glutathione. Ann. Rev. Biochem., 52: 611-660.

Meister, A. and Larsson, A. (1995). Glutathione synthetase deficiency and other disorders of the gamma-glutamyl cycle. In: Scriver, C. R.; Kinzler, K. W. ; Valle, D., et al., eds. The 
Metabolic and molecular bases of inherited diseases. New York: Mc Graw- Hill, 14611477.

Nagaoka, S., Okauchi, Y., Urano, S., Nagashima, U. and Mukai, K. (1990). Kinetic and ab initio study of the prooxidant effect of vitamin E: hydrogen abstraction from fatty acid esters and egg yolk lecithin. J. Am. Chem. Soc., 112:8921-8924.

Nozik-Grayck, E., Suliman, H. and Piantadosi, C. (2005). Extracellular superoxide dismutase. Int. J. Biochem. Cell Biol., 37 (12): 2466-2471.

Oja, S. S., Janaky, R., Varga, V. and Saranasaari, P. (2000): Modulation of glutamate receptor functions by glutathione. Neurochem. Int., 37: 299-306.

Packer, L. (1994). Methods in Enzymology; Oxygen Radicals in Biological Systems, Part C, Academic Press, 1994.

Peake, J. (2003). Vitamin C: Effects of exercise and requirements with training. Int. J. Sport Nutr. Exerc. Metab., 13 (2): 125-151.

Pellegrini, N. Serafini, M., Colombi, B., Del Rio, D., Salvatore, S., Bianchi, M. and Brighenti, F. (2003): Total antioxidant capacity of plant foods, beverages and oils consumed in italy assessed by three different in vitro assays. J. Nutr. 133:2812-2819.

Preston, T.J., Muller. W.J. and Singh, G. (2001): Scavenging of extracellular H2O2 by catalase inhibits the proliferation of HER-2/Neu-transformed Rat-1 fibroblasts through the induction of a stress response. J. Biological Chem., 276: 9558-9564.

Puchau, B., Ángeles, M., Zulet, de Echávarri, A. G., Hermsdorff, H. H. M., and Martínez, J. A., (2009). Dietary total antioxidant capacity: A novel indicator of diet quality in healthy young adults J. Am. Coll. Nutr., 28: 648-656.

Reiter, R. J. (1995). Oxygen radical detoxification processes during aging: the functional importance of melatonin. Aging (Milano), 7: 340-351.

Reiter, R. J. (2003). Melatonin: clinical relevance. Best Pract. Res. Clinic. Endocr. Metabol., 17 (2):273-285.

Reiter, R. J.; Carneiro, R. C. and Oh, C. S. (1997): Melatonin in relation to cellular antioxidiative defense mechanisms. Horm. Metab. Res., 29: 363-372.

Reiter, R. J.,Tan, D. X., Gitto, E., Sainz, R. M., Mayo, J.C., Leon, J., Manchester, L. C.,Vijayalaxm, Kilic, E. and Kilic, U. (2004). Pharmacological utility of melatonin in reducing oxidative cellular and molecular damage. Pol. J. Pharmacol.,56(2):159-170.

Roberts, LJ., Oates, JA. and Linton, MF. (2007). The relationship between dose of vitamin E and suppression of oxidative stress in humans. Free Radic. Biol. Med., 43 (10): 13881393.

Scandalios, J. G., Guan, 1. and Polidoros, A. (1997): Catalase in plants.: Gene structure, properties, regulation and expression In J. G. Scandalios Ed., Oxidative stress and the molecular biology of antioxidant defenses Pp 343-406.

Sener, G.; Paskaloglu, K.; Toklu, H.; Kapucu, C.; Ayanoglu-Dulger, G.; Kacmaz, A. and Sakarcan, A. (2004): Melatonin ameliorates chronic renal failure-induced oxidative organ damage in rats. J Pineal Res., 36(4):232-241. 
Sesso, H.D.; Liu, S.; Gaziano, J.M. and Buring, J. E. (2003): Dietary Lycopene, Tomato-Based Food Products and Cardiovascular Disease in women. J. Nutr., 133: 2336-2341.

Sies, H. (1997). Oxidative stress: Oxidants and antioxidants. Exp. Physiol., 82(2): 291-295.

Tan, D. X., Manchester, L. C.,Burkardt, S., Sainz, R. M., Mayo, J. C., Kohen, R., Shohami, E., Huo, Y. S., Hardeland, R. and Reiter, R. J. (2001). N-acetyl-N-formyl-5methoxyknuramine, a biogenic amine and melatonin metabolite, functions as a potent antioxidant. FASEB. J., 15: 2294-2296.

Terao, M., Kurosaki, M., Saltini, G., Demontis, S., Marini, M., Salmona, M. and Garattini, E. (2000): Cloning of the cDNAs coding for two novel molybdoflavoproteins showing high similarity with aldehyde oxidase and xanthine oxidoreductase. J. Biol. Chem., 275: 30690-30700.

Undeger, U., Giray, B., Zorlu, A. F., Oge, K. and Bacaran, N. (2004). Protective effects of melatonin on the ionizing radiation induced DNA damage in the rat brain. Exp. Toxicol. Pathol., 55: 379-384.

Ustinova, A. A. and Riabinin, V. E. (2003): Effect of chronic gamma-irradiation on lipid peroxidation in CBA mouse blood serum. Radiat. Biol. Radioecol., 43(4):459-463.

Wakatsuki, A.,Okatani, Y., Izumiya, C. and Lkenoue, N. (1999). Melatonin protect against ischemia and reperfusion- induced oxidative lipid and DNA damage in fetal rat brain. J. Pineal Res., 26: 147-152.

Waldeck, A. R. and Stocker, R. (1996). Radical-initiated lipid peroxidation in low density lipoprotein. Insights obtained from kinetic modeling. Chem. Res. Toxicol., 9: 954-964.

Weir, E.K., Archer, S.L. and Reeves, J.T. (1996). Nitric Oxide and Radicals in the Pulmonary Vasculature, Futura Publishing, Armonk, N.Y.

Wilson, J. (1997): Antioxidant defense of the brain: a role for astrocytes (review). Can. J. phys. Pharmacol., 75: 1149 - 1163.

Wright, R. M., Vaitaitis, G. M., Weigel, L.K., Repine, T. B., McManaman, J. L. and Repine, J.E. (1995): Identification of the candidate ALS2 gene at chromosome 2 q33 as a human aldehyde oxidase gene. Redox Report 1:313-321.

Yang, J.; Lam, E. W.; Hammad, H. M.; Oberley, T. D. and Oberley, L. W. (2002): Antioxidant enzyme levels in oral squamous cell carcinoma and normal human oral epithelium. J. Oral Pathol. Med., 31: 71-77.

Yasminch, W. and Theologides, A. (1993). Catalase as a removing scavenger of hydrogen peroxide: a hypothesis. J. Lab. Clin. Med., 122(1): 110-114.

Zatta, P.,Tognon, G. and Carampin, P. (2003). Melatonin prevent free radical formation due to the interaction between beta-amyloid peptides and metal ions [Al(III), $\mathrm{Zn}(\mathrm{II}), \mathrm{Cu}(\mathrm{II})$, Mn(II), Fe(II)]. J. Pineal. Res., 35: 98-103.

Zebger, I.,Snyder, J. W., Andersen, L. K., Poulsen, L., Gao, Z., Lambert, J. D., Kristiansen, U. and Ogilby, P. R. (2004). Direct optical detection of singlet oxygen from a single cell. Photochem. Photobiol., 79(4): 319-322. 
Zelko, I., Mariani, T. and Folz, R. (2002). Superoxide dismutase multigene family: a comparison of the CuZn-SOD (SOD1), Mn-SOD (SOD2), and EC-SOD (SOD3) gene structures, evolution, and expression. Free Radic. Biol. Med., 33 (3): 337-349. 